\title{
Guest Editorial: Evolutionary Advancements in Wireless Software Defined Radio Systems
}

\author{
Sarvpreet Singh $^{1} \cdot$ Marc Adrat $^{1} \cdot$ Dania Marabissi $^{2} \cdot$ Claudio Armani $^{3}$
}

Published online: 18 February 2016

(C) Springer Science+Business Media New York 2016

The field of wireless communications over the years has been constantly making significant advancements in the domain of Software Defined Radios (SDRs). These reconfigurable radios are also capable to learn from their environment like the spectrum and then undertake rapid decisions based on its selfdefined policies leading to the development of wider range of new applications. Thus, new topics and concepts are not only been investigated in the Digital Signal Processing domain but also in the Cognitive Radio domain for an effective and efficient use of the spectrum. Using such technologies with widely accepted international standards like the Software Communications Architecture (SCA) in military software defined radios also requires that they go through the testing and certification processes. Thus, in this Special Issue of the Springer Journal of Signal Processing Systems, all these topics leading towards the evolution of SDRs are discussed. This Special Issue comprises of three sets containing a total of nine articles.

Marc Adrat

marc.adrat@fkie.fraunhofer.de

Sarvpreet Singh

sarvpreet.singh@fkie.fraunhofer.de

Dania Marabissi

Dania.marabissi@unifi.it

Claudio Armani

claudio.armani@selex-es.com

Fraunhofer FKIE, Wachtberg, Germany

2 Università degli Studi di Firenze, Florence, Italy

3 Selex ES, Rome, Italy
The first set of articles put focus on the various cognitive radio technologies like Dynamic Spectrum Access and Spectrum Sensing. The still existing inefficient use of the spectrum is discussed in various SDR research communities. New and innovative ideas are continuously investigated to use the spectrum in the most efficient way in order to meet the ever growing demands of high data throughput. Use of Cognitive Radios is one such way to achieve it. These radios are able to sense the radio environment in order to identify portions of the spectrum that are unused at a specific time or location and are able to reconfigure themselves. Various algorithms and techniques are being investigated to achieve such results in a dynamic environment.

The second set of articles discusses several advanced Digital Signal Processing techniques. All these techniques aim at optimizing operationally relevant criteria. These criteria vary from achieving higher robustness, lower bit error rates, longer communication ranges, and minimal energy consumption to faster deployments. The proofs of the achievements are given either by simulation or by prototype implementation on off-the-shelf low cost hardware.

The third set of articles illustrates the importance test and certification of Software Defined Radios in the military domain using internationally accepted standard like the SCA. Standards like the SCA allow waveforms to be ported easily across heterogeneous platforms. It also provides neatly defined interfaces and Application Program Interfaces (APIs) aimed to increase portability and interoperability of radios. In the final phase of the development of SDRs, efficient ways of testing and agencies providing the certification of standards play a significant role. 


\section{Cognitive Radio Technologies}

The article "Distributed Spectrum Sensing Using Low Cost Hardware" (10.1007/s11265-015-1033-1) written by Stefan Andreas Grönroos, Kristian Nybom, Jerker Björkqvist from Abo Akademi, Turku, Finland and Juhani Hallio, Jani Auranen, Reijo Ekman from Turku University of Applied Sciences, Finland presents a comparison between spectrum sensing performed using a network of nodes build with off the shelf low cost hardware consisting of Raspberry Pi, DVB$\mathrm{T}$ SDR receivers and spectrum sensing performed using a professional sensing system. It shows how the placing of nodes and the antenna quality can influence the end results.

The article "Dynamic Spectrum Access Integrated in a Wideband Cognitive RF-Ethernet Bridge for Industrial Control Applications" (10.1007/s11265-015-1091-4) written by Pedro Manuel Rodríguez Torija, Raul Torrego, Felix Casado, Zaloa Fernandez, Mikel Mendicute, Aitor Arriola, Iñaki Val from IK4-Ikerlan, Mondragon, Spain proposes a DSA scheme based on two spectrum sensing algorithms in a wideband cognitive RF-Ethernet bridge for industrial control applications. It also presents the FPGA implementation of the bridge along with measurement results.

The article "Cognitive Radio as the facilitator for advanced communications electronic warfare solutions" (10.1007/s11265015-1050-0) written by Kresimir Dabcevic, Muhammad Ozair Mughal, Lucio Marcenaro, Carlo S Regazzoni from Universita degli Studi di Genova, Genova, Italy presents the development and implementation of a Spectrum Intelligence algorithm for RF interference mitigation. The aim is to enhance the domain of Electronic Warfare by aiding the development of intelligent self-reconfigurable systems for jamming. The tests are performed using a Cognitive Radio test bed with actual military SDRs.

The article "Design and Experimental Evaluation of a Database-Assisted V2V Communications System over TV White Space" (10.1007/s11265-015-1035-z) written by Onur Altintas, Toshihiko Watanabe, Haris Kremo, Hideaki Tanaka from TOYOTA InfoTechnology Center, Tokyo, Japan and Hitoshi Nakao, Kazuya Tsukamoto, Masato Tsuru from Kyushu Institute of Technology, Tokyo, Japan outlines the general architecture and operation principles of a vehicle-tovehicle communications system over TV white space using both geolocation database and spectrum sensing. It also presents the middleware-centric implementation and test results of a multi-hop vehicle-to-vehicle communications system over the licensed TV-band.

\section{Digital Signal Processing}

The article "Software Defined Radio Implementation of CloudRAN GSM Emergency Service” (10.1007/s11265-015-
1040-2) written by Luca Simone Ronga, Renato Pucci from CNIT, Florence, Italy and Enrico Del Re from University of Florence, Florence, Italy describes an indoor experimental setup where a GSM based cellular network is realized using open source implementation of GSM stack with off the shelf low cost hardware. The focus is to use such implementations for a fast deployment in case of providing an unplanned emergency.

The article "Minimization of wireless sensor network energy consumption through optimal modulation scheme and channel coding strategy" (10.1007/s11265-015-1039-8) written by Rajoua Anane, Kosai Raoof from Laboratory of Acoustics at University of Maine, France and Ridha Bouallegue from Innovation of Communication and Cooperative Mobiles, Innov'COM Lab Sup'Com, Tunisia explores the best modulation strategy to minimize the total energy consumption required to send a given number of bits in a scenario for Wireless Sensor Networks. It also presents a comparative analysis in terms of energy consumption and probability of Bit Error Rate (BER) referring to MSK modulation with proper error control codes approach.

The article "Analysis of BICM-ID Receivers Exploiting Transformations of Extrinsic Information" (10.1007/s11265015-1046-9) written by Marc Adrat, Matthias Tschauner, Markus Antweiler from Fraunhofer FKIE, Germany and Jan Lewandowsky from University of Federal Armed Forces, Munich, Germany presents a novel idea to increase the applicability of modern BICM-ID receivers to legacy waveforms which uses state-of-the-art BICM. It makes use of the so-called "Transformation of Extrinsic Information" to perform a transformation between two different mapping domains based on box-plus and box-minus matrix operations and presents the simulation results in the end.

\section{SCA Test and Certification}

The article "An Approach to T\& E of Military SDR Platforms and Waveforms: The LANCERS Lab" (10.1007/s11265-0151031-3) written by Fulvio Arreghini, Andrea Manco from Istituto per le Telecomunicazioni “G. Vallauri”, Italian Navy, Livorno, Italy and Carmine Vitiello, Marco Luise, Giacomo Bacci from Ingegneria dell'Informazione, University of Pisa, Pisa, Italy, and CNIT, Parma Italy and Matteo Falzarano from Italian Navy General Staff presents an approach for developing a governmental capability for test and evaluation of military SDR platforms and tactical waveforms. This has been named as the "LANCERS Laboratory". Besides the current early development stage, the aim is to merge efforts with the CEVA laboratory regarding security certifications and expand studies towards the SW Defined Radar Sensors (SDRS). 
The article "Achieving SCA Conformance Testing with Model-Based Testing” (10.1007/s11265-015-1089-y) written by Fabien Peureux, Bruno Legeard from University of Franche-Comté, Besançon, France and Julien Botella from SmartTesting, France and Jean-Philippe Delahaye from DGA/ CELAR, French MoD and Eddie Jaffuel from eConsulting, France presents an approach where Model-Based Testing methodology is used to achieve conformance to the SCA standards as described by the JTNC. The conformance tests and scripts are then run on the radio platforms to evaluate the functional conformance of the software implementation with respect to their associated requirements.

Acknowledgements In this Special Issue of the Springer Journal of Signal Processing Systems, evolutionary steps in the wireless communications systems like the software defined radios and cognitive radios are discussed.

As Guest Editors, we express our sincere gratitude and warm thanks to all the reviewers for providing us timely and insightful feedback for this Special Issue. Their unconditional support for the Editorial Board and professionalism towards the authors is highly appreciated. We would also like to thank the team at Springer for their work and support in setting up this Special Issue.

We hope that you will find this Special Issue informative and will assist you towards building a higher learning curve in your work.

Guest Editors:

Sarvpreet Singh

Marc Adrat

Dania Marabissi

Claudio Armani

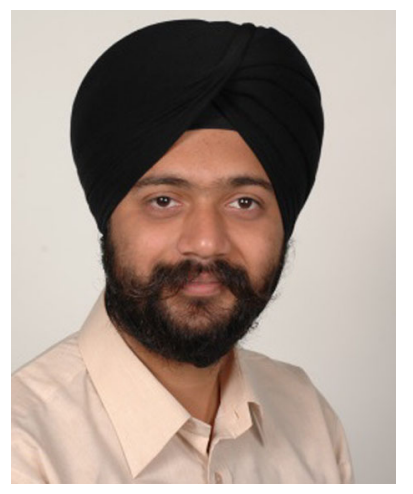

Sarvpreet Singh received his B.E. degree in Computer Science and Engineering from Visvesvaraya Technological University, India in 2003. He received his MSc. degree in Media Informatik from RWTH Aachen University, Germany in 2007. Since July 2007 , he is working as a Research Scientist in the Software Defined Radio Group under the Communications System department of the Fraunhofer Institute for Communication, Information Processing and Ergonomics (FKIE). His current research interests include software defined radio, cognitive radio and waveform development environments focusing on interoperability and portability of waveforms. He has published several technical papers and reports in the above research areas. He has also served as Session Chair, Paper Review Committee Chair and part of Technical Program Committee at several conferences of the Wireless Innovation Forum (WInnF).

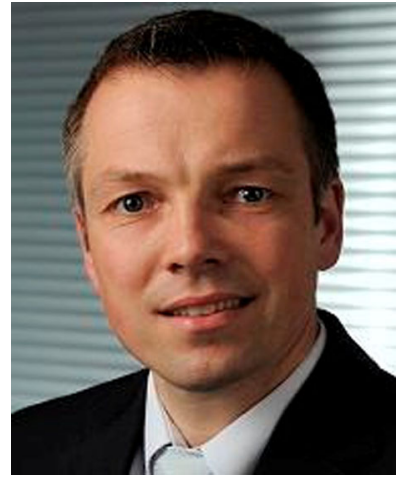

Marc Adrat received his Dipl.Ing. degree in electrical engineering and the Dr.-Ing. degree (PhD) from RWTH Aachen University, Germany, in 1997 and 2003, respectively. Since April 2005, he is with the Fraunhofer Institute for Communication, Information Processing and Ergonomics (FKIE). He is the head of the Software Defined Radio group in the Communication System department. His current research interests include software defined radio, cognitive radio, (military) waveform design as well as concepts for waveform development environments. Matters of particular interest are wideband networking waveforms as well as waveform development environments for portable, interoperable and energy-efficient waveforms. He is in the Board of Directors of the Wireless Innovation Forum (WInnF) and acts as an Advisor for the WInnF's Coordinating Committee for International SCA Standards.

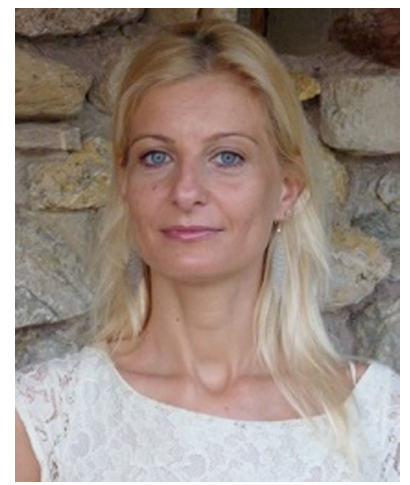

Dania Marabissi received her Master Degree in Telecommunication Engineering summa cum laude in 2000 and the title of $\mathrm{PhD}$ in Computer Engineering and Telecommunications in 2004, from the University of Florence, Italy. In 2000 she joined the Department of Information Engineering, University of Florence, where she currently works as assistant professor. Dania Marabissi is winner of the contest "FIR Futuro in Ricerca 2013" funded by the Italian Ministry of Education. She is also responsible of the scientific activities of the TICom (Technologies for Information and Communication) consortium, born between the University of Florence and Selex ES S.p.A., a Finmeccanica company. She is author of more than 80 publications on journals, international conference proceedings and books and she is owner of an European Patent. She is Associated Editor for the Journal IEEE Transaction on Vehicular Technology and IET Communications journal, member of the Editorial Board of the journal "The Scientific World Journal" Hindawi, Technical Program Committee member for several international conferences, member of the Organizing Committee of the TIWDC 2006 conference, Co-chair of several workshops and Paper Review Committee chair of the SDR- WINN Comm Europe 2014. 


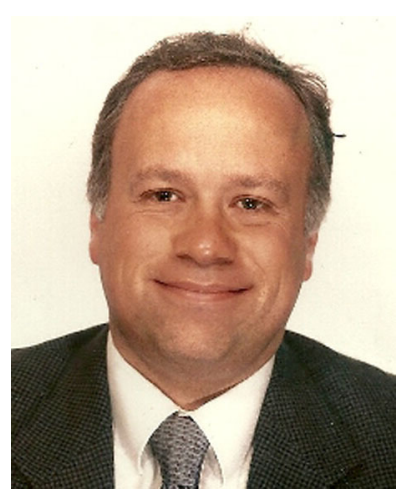

Claudio Armani received his degree in Mathematics and Computer Science summa cum laude from University of Genoa, Italy, in 1988. Since early 2000's, he hold responsibility roles in Wireless Divisions of Multinational Companies focused in Telecommunication Business. Personal focus has always been in identifying system and product solutions starting from the leading research topic of the Telecommunication Industries as Cognitive Radio, Software Defined Radio and modern network-enabled and network-centric wideband meshed waveforms. In Military market, Land \& Naval mobile segment, He is currently accomplishing the role of Capabilities Manager in the CTO organization of the Business Unit in FINMECCANICA company of Italy. He is in the Board of Directors of the Wireless Innovation Forum (WInnF) and acts as a member of the WInnF's Coordinating Committee for International SCA Standards. 